\title{
Experimental characterization of active plasma lensing for electron beams
}

Cite as: Appl. Phys. Lett. 110, 104101 (2017); https://doi.org/10.1063/1.4977894

Submitted: 27 January 2017 . Accepted: 20 February 2017 . Published Online: 06 March 2017

R. Pompili, M. P. Anania, (D) M. Bellaveglia, A. Biagioni, (D) S. Bini, F. Bisesto, E. Brentegani, (D) G. Castorina, (D) E. Chiadroni, A. Cianchi, M. Croia, D. Di Giovenale, M. Ferrario, F. Filippi, A. Giribono, V. Lollo, (D) A. Marocchino, M. Marongiu, A. Mostacci, G. Di Pirro, S. Romeo, A. R. Rossi, J. Scifo, V. Shpakov, C. Vaccarezza, F. Villa, and A. Zigler
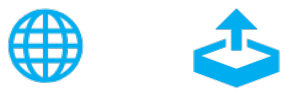

\section{ARTICLES YOU MAY BE INTERESTED IN}

Experimental characterization of the effects induced by passive plasma lens on high brightness electron bunches

Applied Physics Letters 111, 184101 (2017); https://doi.org/10.1063/1.4999010

Comparative study of active plasma lenses in high-quality electron accelerator transport lines Physics of Plasmas 25, 056702 (2018); https://doi.org/10.1063/1.5018001

Guiding of charged particle beams in curved capillary-discharge waveguides

AIP Advances 8, 015326 (2018); https://doi.org/10.1063/1.5011964

\section{Hall Effect Measurement Handbook}

A comprehensive resource for researchers Explore theory, methods, sources of errors, and ways to minimize the effects of errors

(n)

\section{Request it here}

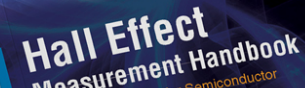

Measurement Hand

Measu

Hakeshore

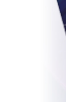




\title{
Experimental characterization of active plasma lensing for electron beams
}

\author{
R. Pompili, ${ }^{1, a)}$ M. P. Anania, ${ }^{1}$ M. Bellaveglia, ${ }^{1}$ A. Biagioni, ${ }^{1}$ S. Bini, ${ }^{1}{ }^{\text {F. B Bisesto, }}{ }^{1}$ \\ E. Brentegani, ${ }^{1}$ G. Castorina,${ }^{1,2}$ E. Chiadroni, ${ }^{1}$ A. Cianchi, ${ }^{3}$ M. Croia, ${ }^{1}$ D. Di Giovenale, ${ }^{1}$ \\ M. Ferrario, ${ }^{1}$ F. Filippi, ${ }^{1}$ A. Giribono, ${ }^{4}$ V. Lollo, ${ }^{1}$ A. Marocchino, ${ }^{1}$ M. Marongiu, ${ }^{4}$ A. Mostacci, ${ }^{4}$ \\ G. Di Pirro, ${ }^{1}$ S. Romeo, ${ }^{1}$ A. R. Rossi, ${ }^{5}$ J. Scifo, ${ }^{1}$ V. Shpakov, ${ }^{1}$ C. Vaccarezza, ${ }^{1}$ F. Villa, ${ }^{1}$ \\ and A. Zigler ${ }^{6}$ \\ ${ }_{1}^{1}$ Laboratori Nazionali di Frascati, Via Enrico Fermi 40, 00044 Frascati, Italy \\ ${ }^{2}$ University of Catania, Piazza Università 2, 95131 Catania, Italy \\ ${ }^{3}$ INFN - Tor Vergata University, Via Ricerca Scientifica 1, 00133 Rome, Italy \\ ${ }_{5}^{4}$ Sapienza University, Piazzale Aldo Moro 5, 00185 Rome, Italy \\ ${ }^{5}$ INFN Milano, via Celoria 16, 20133 Milan, Italy \\ ${ }^{6}$ Racah Institute of Physics, Hebrew University, 91904 Jerusalem, Israel
}

(Received 27 January 2017; accepted 20 February 2017; published online 6 March 2017)

\begin{abstract}
The active plasma lens represents a compact and affordable tool with radially symmetric focusing and field gradients up to several $\mathrm{kT} / \mathrm{m}$. In order to be used as a focusing device, its effects on the particle beam distribution must be well characterized. Here, we present the experimental results obtained by focusing an high-brightness electron beam by means of a $3 \mathrm{~cm}$-long discharge-capillary pre-filled with Hydrogen gas. We achieved minimum spot sizes of $24 \mu \mathrm{m}$ (rms) showing that, during plasma lensing, the beam emittance increases due to nonlinearities in the focusing field. The results have been cross-checked with numerical simulations, showing an excellent agreement.

Published by AIP Publishing. [http://dx.doi.org/10.1063/1.4977894]
\end{abstract}

High-brightness photo-injectors are capable of driving electron beams with high peak current and low emittance as the ones required, for instance, in Plasma Wakefield Acceleration, ${ }^{1,2}$ generation of THz (Ref. 3) and FEL (Free Electron Laser) radiation, ${ }^{4}$ Inverse Compton Scattering ${ }^{5,6}$ and Transmission Electron Microscopy. ${ }^{7}$ In such applications, very dense beams are desired, thus a proper focusing system has to be considered. Permanent-magnet quadrupoles (PMQ) represent a possible approach since they reach focusing gradients up to $560 \mathrm{~T} / \mathrm{m}^{8}{ }^{8}$ However, being the focusing nonsymmetric and the gradient fixed, non-trivial movable systems consisting of at least three lenses are needed in order to produce round beams with adjustable focal length. These issues can be solved by moving toward plasma-based lenses. ${ }^{9-11}$ Tests on electron ${ }^{12}$ and ion ${ }^{13,14}$ beams proved the possibility to achieve much stronger focusing gradients, of the order of $\mathrm{kT} / \mathrm{m}$. Recent results have also been obtained with the so-called "active" plasma lens, demonstrating the focusing of laser-plasma accelerated electrons. ${ }^{15}$ Such device consists of a discharge-capillary that generates an azimuthally symmetric magnetic field whose strength radially increases. Unlike quadrupoles, the active plasma lens focuses simultaneously in both transverse planes.

However, no detailed study of the effects induced on the beam itself has been carried out so far. It represents a crucial point in order to determine the effective usefulness of the active plasma lens. Here, we report its complete characterization performed by means of an high-brightness electron beam with low emittance and very narrow energy spread. The experiment has been carried out at the SPARC_LAB test-facility ${ }^{16}$ with an active plasma lens device consisting of a $3 \mathrm{~cm}$-long discharge-capillary filled by Hydrogen gas. ${ }^{17}$

a)riccardo.pompili@lnf.infn.it
We measured the full 6D beam phase-space (energy, duration and emittance) analyzing the effects introduced by the plasma lensing. Three consecutive screens are employed in order to characterize the beam evolution during its focusing. The results are supported with start-to-end simulations, both for the photo-injector and the discharge-capillary dynamics.

Figure 1 illustrates the experimental setup. The bunch is produced by the SPARC photo-injector, ${ }^{18}$ consisting of a 1.6 cell RF-gun ${ }^{19}$ followed by two accelerating sections embedded by solenoid coils. ${ }^{20}$ The results we report have been obtained with a $50 \mathrm{pC}$ bunch at $126 \mathrm{MeV}$ energy $(50 \mathrm{keV}$ energy spread), $1 \mu \mathrm{m}$ normalized emittance, and 1.1 ps duration, measured with a RF-Deflector device. ${ }^{21}$ For such parameters, the smallest bunch transverse size at the capillary entrance is $\sigma=130 \pm 10 \mu \mathrm{m}$, measured with an Optical Transition Radiation (OTR) screen. All quantities are quoted as rms. The capillary consists of a sapphire hollow tube of $1 \mathrm{~mm}$ diameter with length $L_{c}=30 \mathrm{~mm}$, filled at $1 \mathrm{~Hz}$ rate by $\mathrm{H}_{2}$ gas through two symmetric inlets placed at $L_{c} / 4$ and

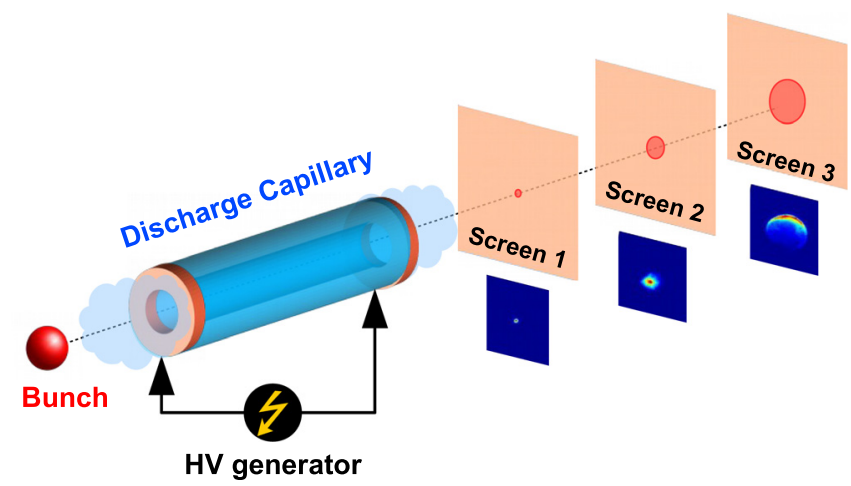

FIG. 1. Experimental layout. The beam passes through the dischargecapillary and is then imaged on the three consecutive screens. The emittance is measured on the last one. 
$3 L_{c} / 4$. The pulsed operation allows maintaining the vacuum level as low as $10^{-8}$ mbar in the RF linac while flowing $\mathrm{H}_{2}$. Two electrodes, placed on each end of the capillary, are connected to a $20 \mathrm{kV}$ generator producing up to $100 \mathrm{~A}$ peak discharge current. ${ }^{22}$ In such conditions, no pinching of the plasma is observed since it would require, for the same capillary, discharge currents of the order of few $\mathrm{kA}^{23,24}$ The entire apparatus is placed in a vacuum chamber directly connected with the last RF section through a windowless, three stage differential pumping system. Since the beam encounters no windows, its emittance is not degraded due to multiple scattering. The beam envelope is measured on three consecutive Ce:YAG screens located 20, 37, and $520 \mathrm{~cm}$ downstream the capillary. The radiation emitted in the forward direction is then collected by a $45^{\circ}$ mirror installed on the same screen holder. The last one is also used for emittance measurements through quadrupole scan, ${ }^{25,26}$ using a triplet of quadrupoles installed upstream. The plasma density is monitored with a Stark broadening-based diagnostics measuring the $\mathrm{H}_{\beta}$ Balmer line. ${ }^{27}$ The apparatus collects the light emitted within $100 \mathrm{~ns}$ along the entire capillary, allowing to reconstruct the longitudinal density profile shown in Fig. 2.

The focusing field produced by the active plasma lens strongly depends on the discharge dynamics along the capillary. In order to describe the main effects of the discharge process, we followed a one-dimensional analytical model described that assumes the distribution of plasma inside the capillary at the equilibrium stage as soon as the discharge is initiated. ${ }^{28}$ Indeed the pinch effect is insignificant for the gas-filled capillary discharge we are describing, therefore we can assume that the equilibrium is determined only by the balance between Ohmic heating and cooling due to the electron heat conduction. We also included in the calculations, the effects arising from the partial ionization of the gas that is produced at low current discharges. The model computes the radial temperature profile $T(r)$ of the plasma in the capillary, allowing retrieving the profile of the current density as $J(r)=\sigma_{e}(r) E$, where $\sigma_{e}(r) \propto T(r)^{3 / 2}$ is the electric conductivity, and $E$ is the electric field associated with the discharge

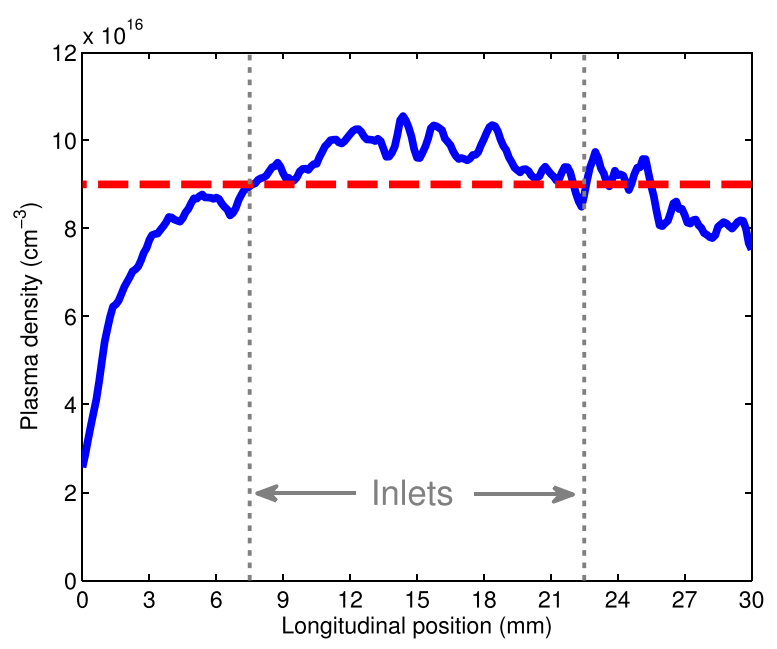

FIG. 2. Measured longitudinal profile of the plasma density averaged over ten shots. The mean value (red line) is $n_{p} \approx 9 \times 10^{16} \mathrm{~cm}^{-3}$. The gray dashed lines refer to the position of the two capillary inlets. current. ${ }^{29}$ Finally the radial profile of the azimuthal magnetic field is obtained from the Ampere law as $B_{\phi}(r)=\left(\mu_{0} / r\right)$ $\int_{0}^{r} J\left(r^{\prime}\right) r^{\prime} d r^{\prime}$. Figure 3 shows the current density (red line) and the resulting magnetic field (blue line) for $I_{D}=45 \mathrm{~A}$ discharge current flowing in the capillary, as used in the experiment. The radial profile has been calculated by using, as input parameters, 40 mbar capillary pressure and $n_{p} \approx 9$ $\times 10^{16} \mathrm{~cm}^{-3}$ average plasma density, as reported in Fig. 2 . The estimated temperature, about $2 \mathrm{eV}$, produces a relatively low ionization of the $\mathrm{H}_{2}$ gas $(\approx 30 \%$ maximum, green line). This strongly affects the whole discharge process, pushing the current to flow mainly on the axis and thus resulting in a radially nonlinear magnetic field. For the sake of completeness, Fig. 3 also shows the simulated bunch transverse profile at capillary entrance (pink line).

The arrival time of the electron beam is scanned with respect to the discharge pulse in order to change the active plasma lens focusing. With the discharge turned off, the unperturbed beam has a spot size $\sigma=110 \pm 6 \mu \mathrm{m}$ as shown in Fig. 4(a). Its emittance is $\epsilon_{n}=1.0 \pm 0.2 \mu \mathrm{m}$, measured by quadrupole scan on the last screen. By turning on the discharge, the beam is focused, but, due to the nonlinear magnetic field as the one calculated in Fig. 3, its emittance becomes larger. At $I_{D} \approx 45 \mathrm{~A}$, corresponding to a focusing gradient of about $100 \mathrm{~T} / \mathrm{m}$, the beam transverse size on the screen is minimized to $\sigma=24 \pm 3 \mu \mathrm{m}$ (Fig. 4(b)) but the corresponding emittance grew up to $\epsilon_{n}=3.6 \pm 0.2 \mu \mathrm{m}$. For larger currents, the beam is over-focused. At $I_{D} \approx 93 \mathrm{~A}$, its focal length is $12 \mathrm{~cm}$, i.e., shorter than the screen distance $(20 \mathrm{~cm})$, thus the resulting spot size appears wider $(\sigma=280 \pm 20 \mu \mathrm{m}$, Fig. 4(c)). In this case, the resulting emittance is $\epsilon_{n}=11$ $\pm 2 \mu \mathrm{m}$. We also investigated the effects of the active plasma lensing on the bunch longitudinal phase-space. For this purpose, we used a fourth screen located downstream the magnetic spectrometer ( $25 \mathrm{keV}$ resolution) with the RF Deflector (30 fs resolution) turned on. The energy spectrum reported in Fig. 4(g) shows that both the central energy and energy spread are actually the same (within 1\%) when the discharge is turned on. The same applies for the bunch temporal profile of Fig. 4(h) indicating that the effects introduced on the beam

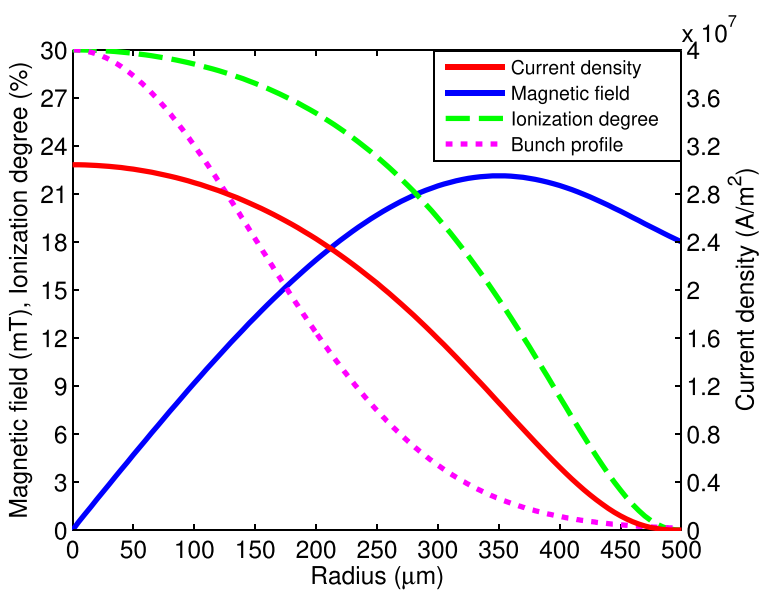

FIG. 3. Calculated radial profiles of the current density (red), azimuthal magnetic field (blue), and $\mathrm{H}_{2}$ ionization degree (green) for $I_{D}=45 \mathrm{~A}$ current discharge. The pink line shows the bunch transverse profile at capillary entrance. 

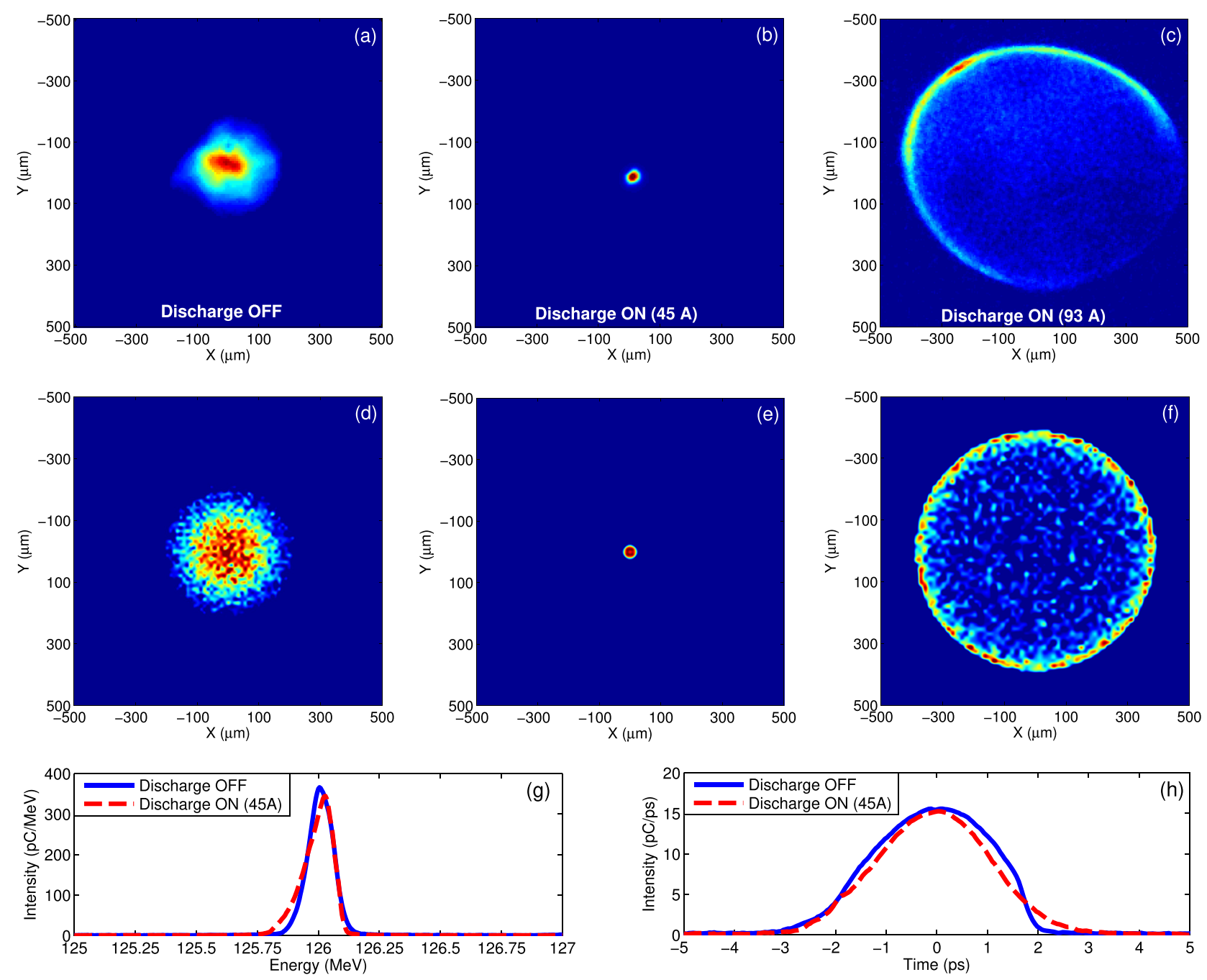

FIG. 4. (a)-(c) Beam spot on the first screen downstream the capillary with the discharge off ( $\sigma \approx 110 \mu \mathrm{m}$, (a)). When turned on, it is $I_{D} \approx 45 \mathrm{~A}$ at $340 \mathrm{~ns}$ delay $\left(\sigma \approx 24 \mu \mathrm{m}\right.$, (b)) and $I_{D} \approx 93 \mathrm{~A}$ at the discharge peak $(\sigma \approx 280 \mu \mathrm{m}$, (c)). (d)-(f) Simulated spot on the same screen. (g) Energy spectrum and (h) temporal profile of the bunch downstream the plasma lens.

phase space are negligible or at least compatible with fluctuations due to RF instabilities.

The complete scan measured on the first screen downstream the capillary at different discharge currents is shown in Fig. 5. The inset also reports the resulting emittance of the beam passing through the capillary before and during the discharge. It is interesting to note the behavior of the beam envelope at later times, when the current is running out. At $I_{D} \approx 15 \mathrm{~A}(\approx 500 \mathrm{~ns}$ after the discharge peak), the beam size does not grow anymore but remains almost stable to $\sigma \approx 50 \mu \mathrm{m}$. This indicates the transition from the active to the so-called passive over-dense plasma lens. ${ }^{12,30}$ Being $n_{b} \approx 10^{12} \mathrm{~cm}^{-3}$ the mean bunch density, in such regime $n_{b} / n_{p} \ll 1$ and the bunch experiences a net focusing gradient equal to $2 \pi e^{2} n_{b} \approx 30 \mathrm{~T} / \mathrm{m}$, i.e., approximately the same due to the active plasma lens at $I_{D} \approx 15 \mathrm{~A}$. Its effect lasts with a plasma recombination, occurring after 7-8 $\mu$ s.

In order to cross-check the experimental results, we performed a start-to-end simulation investigating both the photo-injector and plasma dynamics. The bunch, from its generation to its injection into the capillary, is modeled using the General Particle Tracer (GPT), ${ }^{31}$ while the interaction with the plasma is simulated by Architect, a hybrid-kinetic fluid code. ${ }^{32}$ Figures 4(d)-4(f) shows the simulated beam transverse profiles for several discharge currents. For the plasma lens, we assumed the magnetic focusing field shown in Fig. 3 and $n_{p}=9 \times 10^{16} \mathrm{~cm}^{-3}$ uniform plasma density.

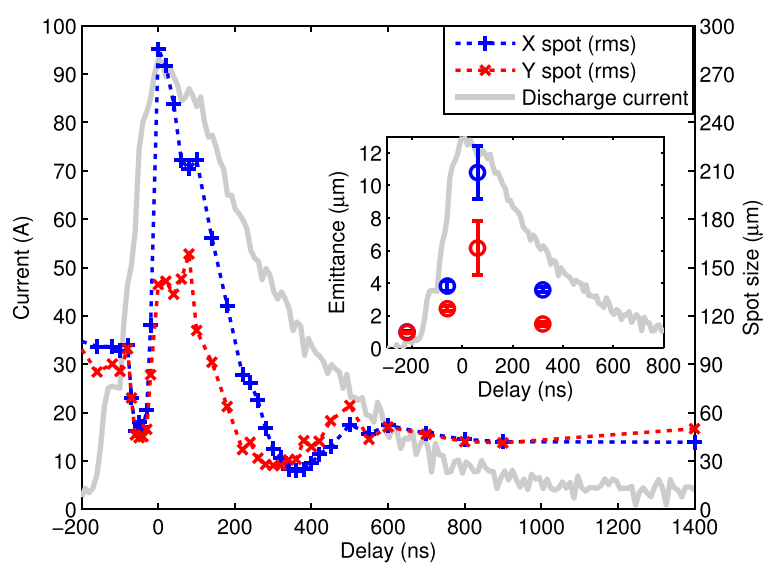

FIG. 5. Envelope scan. The blue (red) points are the measured $\mathrm{X}(\mathrm{Y})$ spot sizes on the first screen downstream the capillary. Each one, obtained at a specific discharge current (gray line), has an associated error of the order of few microns. The blue (red) points in the inset shows the resulting $\mathrm{X}(\mathrm{Y})$ emittance measured at different discharge currents. 

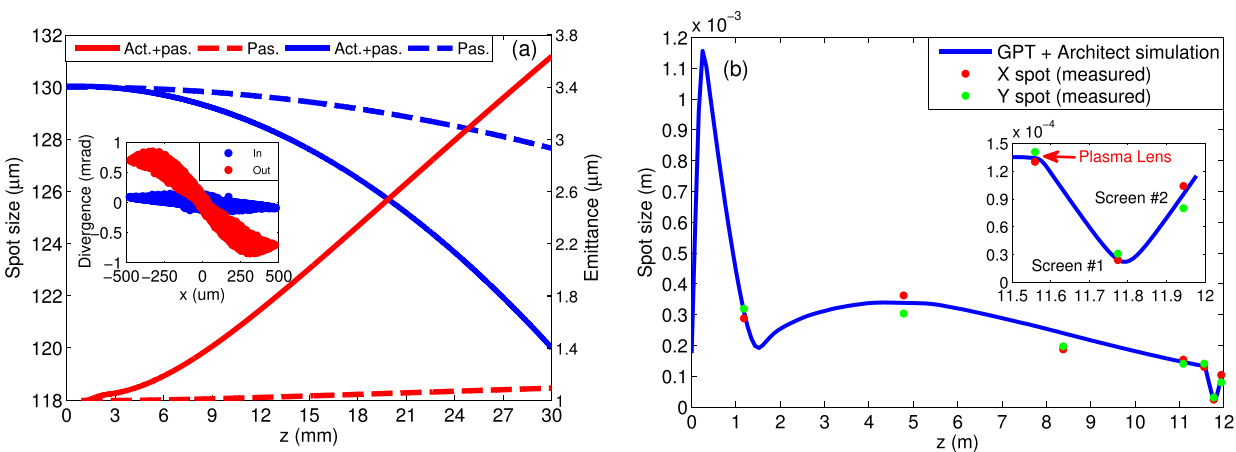

FIG. 6. (a) Simulated envelope (blue) and emittance (red) evolution along the capillary with a plasma density of $n_{p}=9 \times 10^{16} \mathrm{~cm}^{-3}$. The magnetic focusing field for the active plasma lens regime is the one calculated in Fig. 3. The inset shows the trace space at entrance (blue) and exit (red) of the active plasma lens. (b) Envelope along the photo-injector with discharge turned on at $45 \mathrm{~A}$. The blue line represents the simulated beam while the red (green) points refer to measured $\mathrm{X}(\mathrm{Y})$ spot sizes.
Since $n_{b} / n_{p} \ll 1$ along the entire plasma channel (see Fig. 2 ), the excited wakefields are negligible (less than $0.3 \mathrm{MV} /$ $\mathrm{m}$ ) and the longitudinal dynamics is not affected while the bunch propagates through the plasma. ${ }^{33}$ This confirms our experimental findings reported in Figs. 4(g) and 4(h). On the contrary, severe effects are introduced in the transverse plane by the nonlinear focusing field that induces the spherical aberrations shown in Fig. 4(c) and simulated in Fig. 4(f). These are in turn responsible for the emittance growth shown in Fig. 6(a), where the simulation predicts $3.7 \mu \mathrm{m}$ as the final emittance in excellent agreement with the one experimentally measured. The plot also reports the different contributions due to the passive plasma lensing. As one can see, it only produces a slight increase of the beam emittance. Fig. 6(b) shows the complete simulated beam envelope obtained by combining the GPT and Architect results. It can be directly compared with the experimental one measured by using the several screens placed along the photo-injector.

In conclusion, we have presented an experimental characterization of an active plasma lens consisting of a $3 \mathrm{~cm}$-long discharge-capillary. Such device represents a reliable and affordable solution because of its compactness, strong focusing, and easy tunability. We have investigated its effects on a highbrightness electron beam for different values of the discharge current. Results demonstrate that the active plasma lens allows to reach a minimum waist of $24 \mu \mathrm{m}$ with $20 \mathrm{~cm}$ focal length, i.e., about five times smaller than the unfocused beam. We have also found that the magnetic focusing field, when operating at low discharge currents, can be highly nonlinear at an increasing radii, leading to an emittance increase due to spherical aberrations. It is most likely due to the low partial ionization reached in the $\mathrm{H}_{2}$ gas that in turn is dictated by the relatively low discharge current $(\approx 45 \mathrm{~A})$ employed to focus the beam on such location. Several setups, involving different capillary geometries and discharge circuits, are currently under study with the goal to improve the overall quality of the focused beam. Our results, however, provide a systematic study of the focusing properties of the active plasma lens and represent a first step toward the full optimization of such a device.

This work has been partially supported by the EU Commission in the Seventh Framework Program, Grant Agreement No. 312453-EuCARD-2 and the Italian Research Minister in the framework of FIRB - Fondo per gli Investimenti della Ricerca di Base, Project No. RBFR12NK5K. We wish to acknowledge Stefano Pella for his support in the development of the discharge device.
${ }^{1}$ P. Chen, J. Dawson, R. W. Huff, and T. Katsouleas, Phys. Rev. Lett. 54, 693 (1985).

${ }^{2}$ M. Litos, E. Adli, W. An, C. Clarke, C. Clayton, S. Corde, J. Delahaye, R. England, A. Fisher, J. Frederico et al., Nature 515, 92 (2014).

${ }^{3}$ F. Giorgianni, E. Chiadroni, A. Rovere, M. Cestelli-Guidi, A. Perucchi, M. Bellaveglia, M. Castellano, D. Di Giovenale, G. Di Pirro, M. Ferrario et al., Nat. Commun. 7, 11421 (2016).

${ }^{4}$ V. Petrillo, M. Anania, M. Artioli, A. Bacci, M. Bellaveglia, E. Chiadroni, A. Cianchi, F. Ciocci, G. Dattoli, D. Di Giovenale et al., Phys. Rev. Lett. 111, 114802 (2013).

${ }^{5}$ R. W. Schoenlein, W. Leemans, A. Chin, and P. Volfbeyn, Science 274, 236 (1996).

${ }^{6}$ A. Bacci, D. Alesini, P. Antici, M. Bellaveglia, R. Boni, E. Chiadroni, A. Cianchi, C. Curatolo, G. Di Pirro, A. Esposito et al., J. Appl. Phys. 113, 194508 (2013).

${ }^{7}$ R. Li and P. Musumeci, Phys. Rev. Appl. 2, 024003 (2014).

${ }^{8}$ J. Lim, P. Frigola, G. Travish, J. Rosenzweig, S. Anderson, W. Brown, J. Jacob, C. Robbins, and A. Tremaine, Phys. Rev. Spec. Top.-Accel. Beams 8, 072401 (2005).

${ }^{9}$ P. Chen, K. Oide, A. Sessler, and S. Yu, Phys. Rev. Lett. 64, 1231 (1990).

${ }^{10}$ J. Rosenzweig, B. Breizman, T. Katsouleas, and J. Su, Phys. Rev. A 44, R6189 (1991).

${ }^{11}$ H. Nakanishi, Y. Yoshida, T. Ueda, T. Kozawa, H. Shibata, K. Nakajima, T. Kurihara, N. Yugami, Y. Nishida, T. Kobayashi et al., Phys. Rev. Lett. 66, 1870 (1991)

${ }^{12}$ G. Hairapetian, P. Davis, C. Clayton, C. Joshi, S. Hartman, C. Pellegrini, and T. Katsouleas, Phys. Rev. Lett. 72, 2403 (1994).

${ }^{13}$ W. K. H. Panofsky and W. Baker, Rev. Sci. Instrum. 21, 445 (1950).

${ }^{14}$ E. Boggasch, A. Tauschwitz, H. Wahl, K.-G. Dietrich, D. Hoffmann, W. Laux, M. Stetter, and R. Tkotz, Appl. Phys. Lett. 60, 2475 (1992).

${ }^{15}$ J. Van Tilborg, S. Steinke, C. Geddes, N. Matlis, B. Shaw, A. Gonsalves, J. Huijts, K. Nakamura, J. Daniels, C. Schroeder et al., Phys. Rev. Lett. 115, 184802 (2015).

${ }^{16}$ M. Ferrario, D. Alesini, M. Anania, A. Bacci, M. Bellaveglia, O. Bogdanov, R. Boni, M. Castellano, E. Chiadroni, A. Cianchi et al., Nucl. Instrum. Methods B 309, 183 (2013).

${ }^{17}$ A. Biagioni, M. Anania, M. Bellaveglia, E. Chiadroni, A. Cianchi, D. Di Giovenale, G. Di Pirro, M. Ferrario, F. Filippi, A. Mostacci et al., J. Instrum. 11, C08003 (2016).

${ }^{18}$ D. Alesini, S. Bertolucci, M. Biagini, C. Biscari, R. Boni, M. Boscolo, M. Castellano, A. Clozza, G. Di Pirro, A. Drago et al., Nucl. Instrum. Methods A 507, 345 (2003)

${ }^{19}$ A. Cianchi, D. Alesini, A. Bacci, M. Bellaveglia, R. Boni, M. Boscolo, M. Castellano, L. Catani, E. Chiadroni, S. Cialdi et al., Phys. Rev. Spec. Top.Accel. Beams 11, 032801 (2008).

${ }^{20}$ M. Ferrario, D. Alesini, A. Bacci, M. Bellaveglia, R. Boni, M. Boscolo, M. Castellano, E. Chiadroni, A. Cianchi, L. Cultrera et al., Phys. Rev. Lett. 104, 054801 (2010).

${ }^{21}$ D. Alesini, G. Di Pirro, L. Ficcadenti, A. Mostacci, L. Palumbo, J. Rosenzweig, and C. Vaccarezza, Nucl. Instrum. Methods Phys. Res. Sect. A 568, 488 (2006).

${ }^{22}$ M. Anania, A. Biagioni, E. Chiadroni, A. Cianchi, M. Croia, A. Curcio, D. Di Giovenale, G. Di Pirro, F. Filippi, A. Ghigo et al., Nucl. Instrum. Methods Phys. Res. Sect. A 829, 254 (2016).

${ }^{23}$ N. Bobrova, S. Bulanov, D. Farina, R. Pozzoli, T. Razinkova, J. Sakai, P. Sasorov, and I. Sokolov, Laser Part. Beams 18, 623 (2000).

${ }^{24}$ D. Spence, A. Butler, and S. Hooker, JOSA B 20, 138 (2003). 
${ }^{25}$ A. Mostacci, M. Bellaveglia, E. Chiadroni, A. Cianchi, M. Ferrario, D. Filippetto, G. Gatti, and C. Ronsivalle, Phys. Rev. Spec. Top.-Accel. Beams 15, 082802 (2012).

${ }^{26}$ A. Cianchi, D. Alesini, M. Anania, A. Bacci, M. Bellaveglia, M. Castellano, E. Chiadroni, D. Di Giovenale, G. Di Pirro, M. Ferrario et al., Phys. Rev. Spec. Top.-Accel. Beams 18, 082804 (2015).

${ }^{27}$ F. Filippi, M. Anania, A. Biagioni, E. Chiadroni, A. Cianchi, M. Ferrario, A. Mostacci, L. Palumbo, and A. Zigler, J. Instrum. 11, C09015 (2016).

${ }^{28}$ N. Bobrova, A. Esaulov, J.-I. Sakai, P. Sasorov, D. Spence, A. Butler, S. Hooker, and S. Bulanov, Phys. Rev. E 65, 016407 (2001).
${ }^{29}$ S. Eliezer, The Interaction of High-Power Lasers with Plasmas (CRC Press, 2002).

${ }^{30}$ J. Su, T. Katsouleas, J. Dawson, and R. Fedele, Phys. Rev. A 41, 3321 (1990).

${ }^{31}$ M. De Loos and S. Van der Geer, in 5th European Particle Accelerator Conference (1996), p. 1241.

${ }^{32}$ A. Marocchino, F. Massimo, A. Rossi, E. Chiadroni, and M. Ferrario, Nucl. Instrum. Methods Phys. Res. Sect. A 829, 386 (2016).

${ }^{33}$ W. Lu, C. Huang, M. Zhou, W. Mori, and T. Katsouleas, Phys. Plasmas 12, 063101 (2005). 